ORIGINAL ARTICLE

\title{
Prevalence of Sarcopenia and Its Association with Socioeconomic Status among the Elderly in Tehran
}

\author{
Ahmadreza Dorosty ${ }^{1}$, Godana Arero ${ }^{2,3}$, Maryam Chamar ${ }^{1}$, Sogand Tavakoli ${ }^{1}$
}

ABSTRACT

BACKGROUND: Sarcopenia is a syndrome characterized by progressive and generalized loss of skeletal muscle mass and strength. It imposes significant costs on health care systems. Socioeconomic status is also the root cause of healthy challenges among the elderly. Therefore, investigating the association between sarcopenia and socioeconomic status is very important to improve healthy ageing of the elderly. The aim of this study was to investigate the prevalence of sarcopenia and its association with socioeconomic status among the elderly in Tehran.

METHODS: Cross-sectional and case-control studies were conducted from August 2014-July 2015 among 310 men and 334 women elderly (60 and over years old) in Tehran health centers. Randomization, restriction and matching were setting during study design to minimize selection bias. Then study participants were recruited via phone call. Participants' phone numbers were already recorded in a telephone book electronically. When there were two elderly people in the same house, only one person was invited randomly. Association between sarcopenia and socio-economic status was analyzed by SPSS version 22.

RESULTS: The overall prevalence of sarcopenia in the elderly was $16.5 \%$. Prevalenceamong the lowincome elderly was relatively higher than (20.5\%) that among those with middle income status (18.2\%) while in the higher income, the proportion of sarcopenia was very low (12.8\%). The findings indicated that 339(52.6\%) were in low-income status, 304(47.1\%) were in middle-income status and $1(.2 \%)$ in high-income class.

CONCLUSION: There was a significant association between socioeconomic status and sarcopenia (Pvalue $<0.001$. The odd risk of sarcopenia was 0.97 times more likely higher in low socioeconomic class than those who were in middle and high income classes.

KEYWORDS: Sarcopenia, Socioeconomic status, elderly people

DOI: http://dx.doi.org/10.4314/ejhs.v26i4.11

\section{INTRODUCTION}

Sarcopenia is a syndrome characterised by progressive and generalised loss of skeletal muscle mass and strength with a risk of adverse outcomes such as physical disability, poor quality of life and death $(1,2)$. It was first coined as "sarcopenia" by Irwin Rosenberg $(3,4)$ in 1998. More recently, on the third updated published article, in 2014, the European Working Group on Sarcopenia (EWGSOP) again recognized that the key element is a loss of muscle strength (dynapenia) rather than a loss of muscle mass (5). This has led to a change in the definition of sarcopenia to include strength (grip strength) or function (walking speed or distance). Now, sarcopenia is defined as a decline in walking speed or grip strength associated with low muscle mass. Based on this concept, a number of societies around the world have provided revised definitions of sarcopenia (6-11). These definitions have to some extent deemphasized the importance of aging, recognizing that sarcopenia has a variety of causes in addition to physiological

\footnotetext{
${ }^{1}$ Department of Community Nutrition, School of Nutritional Sciences \& dietetics, Tehran University of Medical Sciences, Tehran, Iran

${ }^{2}$ Department of Epidemiology and Biostatistics School of Public Health,Tehran University of Medical Sciences, Tehran, Iran

${ }^{3}$ Tehran University of Medical Sciences, International Campus ( TUMS-IC), Tehran, Iran

Corresponding Author: Godana Arero, Email: garero2015@gmail.com
} 
effects of aging (11).

Sarcopenia imposes significant costs on health care systems. In the United States alone, sarcopenia-related costs were estimated to be more than $\$ 18.5$ billion in 2000 (12). Sarcopenia is considered to be the underlying cause of frailty (13), which is in turn the sixth cause of death among people over 65 years old. It also increases the risk of falling and disability among the elderly (13). The modifiable behavioral factors such as physical activity level, androgen hormone level (14), smoking habits and, particularly, the quality and quantity of dietary intake (15) can be important in delaying or even preventing sarcopenia. In addition, socioeconomic status is the root cause of health issues among the elderly (16). There are obvious differences between income and economic status and health (17). There are also obvious disparities between the health statuses of the elderly (18). Many studies have investigated the association between nutritient intake such as protein (19-26), vitamins $(27,28)$, minerals (29) and antioxidants (30) and sarcopenia. Our study is a first one in the world which has investigated the association between sarcopenia and socioeconomic status among theelderly. The aim of this study is to assess the association between the prevalence of sarcopenia and socioeconomic status and among the elderly in Tehran.

\section{METHODS AND MATERIALS}

Study design: A study of cross-sectional that began in August 2014 with a probabilistic sampling followed by a case-control study among 644 elderly aged 60 years and older living in the southern part of the Tehran till July 2015 for 13 months. The sociodemographic characteristics included age, gender, marital status; income and schooling were collected through self report. Age was grouped in three 10-year categories, with all those aged 80 years or older combined into one group. Marital status was classified as married (married or in a stable relationship) or not married/single (divorced, separated or widowed). Schooling (in years) was analyzed as a continuous variable.Sampling technique: A pilot study was conducted to validate the procedures at first. We then identified sarcopenic cases among the elderly in the first phase of the study (cross-sectional). Next, sarcopenia cases were assigned for second phase of study (casecontrol) to compare outcomes with socioeconomic status. Each socioeconomic data was first prearranged, categorical and examined alone in order to measure its outcomes with the proportion of the sarcopenia. Group matching was done instead of individual matching by their age, sex and place of residence (ratio was 1:2). Hence, it was very difficult both in time and financial going to find out an accurate listing of all theoretical elderly population in Iran,we should make a difference between the population, we would like to study to, and it was the accessible elderly population in Tehran.The theoretical elderly population in Iran was 6,162,273 (census 2011) while accessible elderly in Tehran was approximately 668,300 (calculated from 2011 census. Then we had randomely selected southern part of Tehran and invited people who able to come to public health centers under the patronage of Tehran University of Medical Sciences. This about one third of public health centers (10/31) located in southern of Tehran. Then through sampling frame which was "electronic telephone books address" we had randomely picked their phone number and invited them to the centers. The participants' phone numbers were already recorded on telephone book/registration book at the nearby health center. Then, the study participants were recruited via phone call to come to the nearby cluster. When there were two eligible elderly people (husband and wife) in a household, only one person was invited randomly to participate in the study. In the case of no eligible person in the selected household, the interviewer would take the next phone call. By doing so, finally, we drew our sample, 644 elderly people from 6 Districts in Tehran, using simple random sampling procedure in the 6 clustered health centers. The sample size was determined using single proportion formula for cross-sectional studies while in case-control, it was determined by comparing two population estimation $\mathrm{P}_{1}$ and $\mathrm{P}_{2}$ (given a $95 \%$ confidence level and $80 \%$ power). To minimize bias, errors and possible confounding 
factors, we randomized, restricted and matched ubjects during study design and data collection. Missing data were checked and controlled by running descriptive frequencies and excluding case pairwise. Therefore, there were no missing data identified during data analysis.

Instruments: Standard questionnaire was used to collect data on income, occupation, education level and health status. To collect socioeconomic data, we used self-report and "household economic based indicators" which include seven items such as a flat television, fridge, carpenter, house, car, laptop and sofa. This questionnaire was taken from the previous a study and standardized in the Iranian context. This questionnaire contains a series of questions about information on a wide range of qualifications including vocational qualifications gained at work place and other household durables or facilities. Respondents that lacked an amenity were asked the reason for not having it. Respondents who answered more than 6 questions and over were considered as a high income status while those who ticked between 3-5 questions were considered as middle income class and those who answered 12 taken as low-income status.

Muscle mass data were taken, measured and calculated from anthropometric variables such as height, hip, waist, wrist, ankle, knee, arm, thigh, upper and median (in meter) and then were entered into the software and analyzed by Bioimpedance analysis (BIA). Other measurements such as weight (in $\mathrm{Kg}$ ) and body mass index were calculated and entered into the same software. Handgrip strength was measured with maximum voluntary contractions for each participant by squeeze bulb dynamometer (c748902 ). We would repeat the measurement three times for each hand with a 30-second rest time in between each trial. Physical performance was measured by doing a 6-meter course gait speed test. Each participant was asked to walk at his/her usual pace to the other end of the 6-meter course. Time was recorded by chronometer in seconds. The cutoff points for each of them were obtained from the previous study. Muscle mass less than $8.87 \mathrm{~kg} / \mathrm{m}^{2}$ for men and $6.42 \mathrm{~kg} / \mathrm{m}^{2}$ for women was considered as abnormal (2, 31-34), while handgrip strength $<30 \mathrm{~kg}$ for men and $<20 \mathrm{~kg}$ for women was taken as abnormal (1) and gait speed $<0.8 \mathrm{~m} / \mathrm{s}$ was abnormal for both genders $(35-37)$.
We used EWGSOP definition of sarcopenia to define the case group. According to this definition, individuals with abnormal muscle mass or abnormal hand grip strength, or weak physical performance were considered as pre-sarcopenic while sarcopenic person was an individual who abnormal was with two variables together (muscle mass and hand grip strength or muscle mass and physical performance or hand grip and physical performance). Those with all the three abnormal criteria were considered as the severely sarcopenic (4). The relationship between sarcopenia and socioeconomic status were analyzed by SPSS software.

Subjects: A total of 644 individuals participated in this study, out of whom $310(41.1 \%)$ and $334(51.9 \%)$ were elderly males and females respectively.

\section{Inclusion criteria:}

- Participants had to be 60 years and above.

- Participants had to be able move without crutches, walker or other assistive devices.

- There had to be an absence of artificial limbs or limb prosthesis.

- There had to be an absence of active cancer, according to individual self report.

- There had be an absence of chronic diseases.

\section{Statistical applications}

Pre-coded data were entered into the computer and cleaned through the phase-by-phase screening using SPSS version 22.0 software for analysis. Multiple analyses such as crosstab, bivariate, independent-sample t-test and logistic regression were used. Frequency, proportion and summary statistics were used to describe the characteristics of the study population. Ten percent $(10 \%)$ of the total data were randomly selected and crosschecked for reliability respective to the original data. The odds ratio and $95 \% \mathrm{CI}$ were computed to see the presence and degree of association between scorpion and socioeconomic status. Pvalue $<0.05$ was considered as statistically significant, and multivariate logistic regression was employed to control possible confounding factors.

Ethical issues: The Ethics Committee and Vice Chancellor for Research at Tehran University of Medical Sciences approved the project in accordance with the tenets of the Helsinki 
Declaration and the national ethical guideline for medical research. The ethical approval code is IR.TUMS.REC.1394.346. Furthermore, informed consent was obtained from the study participants and concerned bodies.

\section{RESULTS}

Table 1 shows general demographic characteristics of the study participants. A total of 644 individuals participated in this research study, out of whom $310(41.1 \%$ ) and 334(51.9\%) were elderly males and females respectively. The mean age of the participants was $(70.8 \pm 6.1)$. There was no case of dropout throughout the study and so the response rate was $100 \%$. The findings of our study indicated that $339(52.6 \%)$ were in lowincome status, $304(47.1 \%)$ were in middle income status and $1(.2 \%)$ in high income class. Using Asian Working Group and European Working Group, the prevalence of sarcopenia was found to be $16.5 \%$ and $32.5 \%$ respectively. Prevalence of pre-sarcopenia was (21.2\%). Severe sarcopenia was not reported during the data collection period. Prevalence of sarcopenia was $27.1 \%$ and $6.6 \%$ among men and women respectively. In crosstab analysis, proportion of pre-sarcopenia and sarcopenia in low-income were a relatively higher than $(22.6 \%$ and $20.5 \%)$ in those with middle income (21.7 and $18.2 \%$ ) while, in the higher income, the proportions of presarcopenia and sarcopenia were lower (13.7 and $12.8 \%)$. Educational level, occupation, and income status largely contributed to the development of sarcopenia among the study participants. For instance, proportion of sarcopenia in an illiterate, primary school and diploma+ were $18.6 \%, 16.2 \%$, and $12.3 \%$ respectively. For those who had occupations, the proportion of sarcopenia was smaller (16.4\%), while, for those who had no occupation, the proportion was relatively high (25\%).
Table 1: General characteristics of study participants

\begin{tabular}{ll}
\hline Variables & $\begin{array}{l}\text { Mean } \pm \text { Standard } \\
\text { deviation }\end{array}$ \\
\hline Age: & $(70.8 \pm 6.1)$ years \\
Sex: & $310(41.1 \%)$ \\
Male & $334(51.9)$ \\
Female & \\
Marital status: & $397(61.6 \%)$ \\
Married & $237(36.7 \%)$ \\
Singles & $6(.9 \%)$ \\
Widower & $296(46.0 \%)$ \\
Divorced & $31.1 \pm 4.7$ \\
BMI (kg/m2) & \\
Educational level: & $296(46.0 \%)$ \\
Illiterate & $210(32.6 \%)$ \\
Primary school & $42(6.5 \%)$ \\
Secondary high school & \\
Diploma, BSC, MSC, & \\
MD & $42(6.5 \%)$ \\
Occupation: & \\
Yes & $4(.6 \%)$ \\
no & $640(99.4 \%)$ \\
\hline
\end{tabular}

Our findings indicated a significant association between sarcopenia and socioeconomic status (pvalue < 0.01). The relationship between socioeconomic status with sarcopenic and the non - scorpion was also investigated using Pearson product-moment correlation coefficient. Preliminary analysis was conducted to ensure no violation of the hypothesis of normality, linearity, and homoscedasticity. The same results were also observed with crosstab analysis that correlation between socioeconomic status and sarcopenia was significant (or $=1, \mathrm{~N}=644$, $\mathrm{P}$-value <0.001). An independent-samples t-test was also performed to study the association between sarcopenic mean scores and socioeconomic status in sarcopenia.

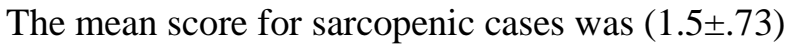
while the mean score for non-sarcopenic group was $(1.60 \pm .67) ; \mathrm{t}(642)=1.79, \mathrm{P}$-value $=0.02$, two-tailed). The magnitude of the differences in the means (mean difference $=0.1,95 \% \mathrm{Cl}: .1-.2$ ) was very small (eta squared $=0.004$ ). The odd risk was calculated from the hypothetical data in table1 
estimated that sarcopenia risk was 0.97 times more likely to develop in those who were in lower socioeconomic status than those who were in middle income class. The number of high income status was negligible. Binary logistic regression was also performed to assess the odd risk of sarcopenia among the low, middle and high income elderly. The model contains five independent variables (sex, age, educational level, occupation, and socioeconomic status). Unadjusted estimates in the model I showed the odd risk of gender was 0.21 , and statistically significant association was observed (P-value $<0.001$, with $95 \% \mathrm{CI}=0.44-1.07$. All other variables were equal.

Table 2. Cross tabulation of socioeconomic \& Sarcopenic group among elderly in Tehran, Iran 2016 (N $=644)$

\begin{tabular}{lccc}
\hline & Low income & Middle \& high income & Total \\
\hline Sarcopenic & 40 & 180 & 220 \\
Non-sarcopenic & 66 & 458 & 524 \\
Total & 106 & 538 & 644 \\
\hline OR & $40 * 458=$ & 0.97 & \\
\cline { 2 - 4 } & $66 * 180$ & &
\end{tabular}

*The odd risk of sarcopenia in low socio-economic class was .97 times higher than those in middle and high income class

Table 3: Prevalence of sarcopenia based on different diagnostic criteria

\begin{tabular}{ll}
\hline Criteria & Prevalence (\%) \\
\hline European Working Group on Sarcopenia (EWGSOP) & $207(32.5)$ \\
Asian Working Group for Sarcopenia (AWGS) & $106(16.5)$ \\
Foundation for the National Institutes of Health (NHI) & $106(16.1)$ \\
\hline
\end{tabular}

\section{DISCUSSION}

The findings of this study indicated that the prevalence of sarcopenia was 16.5 and $32.5 \%$, respectively using different definitions of AWGOS and EWGOS (Table 3). Males had $21.3 \%$ sarcopenic cases compared to $8.9 \%$ in females. This result is found to be higher than a similar study conducted in the USA, in Taiwan and in Hong Kong, which showed the prevalence of sarcopenia as $26.8 \%$ (32), $23.6 \%$ (38) and $12.3 \%$ (39) respectively. Unlike a similar study conducted in New Mexico, which indicated the prevalence of sarcopenia in male elderly to be $50 \%$ (40) and 52\% among male elders in the USA (41), in the present finding, sarcopenia is found to be less prevalent. In a study conducted in Taiwan, the prevalence of sarcopenia was reported as $18.6 \%$ in elderly women and 23.6 in erderly men (36), while in our study, the prevalence of sarcopenia was $8.9 \%$ in elderly women and
$21.3 \%$ in elderly men. This shows that the prevalence in elderly women was higher than our finding in elderly women and relatively in line with elderly men. According to a study conducted by the European Working Group on Sarcopenia (EWGSOP), the prevalence of sarcopenia in elderly females living in community-dwelling and men living in long-term care institutions were $30 \%$ and $68 \%$ respectively $(42,43)$, while our findings showed substantially lower proportion of sarcopenia in both males and females $(21.3 \%$ and $8.9 \%)$. On the other hand, a separate study was conducted in Geriatric hospitalized patients in $\mathrm{J}$ Am Med Dir Association, USA, and communitydwelling persons in Italy who had low muscle mass which indicated that the percentage of sarcopenia scores among the elderly population were $25 \%$ and $20 \%(42,43)$. This finding is higher than the prevalence of our finding which is $32.5 \%$. This study is found to be the first one in the gobe to study the association between sarcopenia and 
socioeconomic status among elderly people. Our results indicated that in low-income people, the prevalence of pre-sarcopenia and sarcopenia were higher $(22.6 \%$ and $20.5 \%)$ than those middle income (21.7 and 18.2\%). In higher income elderly the prevalence of presarcopenia and sarcopenia was very lowe (13.7 and $12.8 \%)$ as compare to low and middle income people. Therefore, as people become poor, the chance of developing sarcopenia is gets high. Also, the findings show that educational level, occupation and income status are the main contributors in developing sarcopenia among study participants. For instance, the proportions of sarcopenia in the uneducated, primary schoolers and diploma holders and those who had above diploma were $18.6 \%, 16.2 \%$, and $12.3 \%$ respectively. In the meantime, having an occupation and good income also played a great role in the development of sarcopenia. Moreover, the findings indicated that the proportion of sarcopenia in elderly people who did not ave any occupation (25\%) was higher than those who had occupations (16.4\%). In addition, the results of the study indicated that the mean scores of sarcopenic people were a bit higher among people with middle and high income status when comparing to non-sarcopenic elderly people $(1.60 \pm 0.67)$ and for non-sarcopenic $(1.5 \pm 0.73)$, $\mathrm{t}(642)=1.79, \mathrm{p}$-value $=0.02$, two-tailed). The findings revealed that in low-income elderly, the chance of developing sarcopenia was higher with an odd risk of 0.97 in low income and 0.8 for both middle and high income elderly persons. These indicate that elderly people who are in low-income status are 0.97 times more likely to develop sarcopenia than those who are in middle or high income status. As mentioned before, this study is the first one in the world to investigate the association between scorpenia and socioeconomic status in elderly people. This makes it difficult to compare our results with another study. On the other hand, a pilot study was conducted before commencement the main study to validate the scale's reliability and validity. Daily field supervision and data checking took place as a follow-up method. We concluded that there is a significant association between sarcopenia and socioeconomic status. Elderly people with lower socioeconomic status are more likely to develop sarcopenia $(\mathrm{p}$-value <.01). Therefore, elderly with these characteristics should be the target for prevention strategies.

\section{ACKNOWLEDGEMENTS}

First of all, I praise "The Name of Almighty GOD" who secures me all the time in all aspects of my life. Next, I would like to acknowledge the Office of the Vice Chancellor for Global Strategies and International Affairs for funding this project [grant number: 93/sc/48/129]. I would also like to acknowledge the School of Nutritional Sciences and Dietetics which supported this project by providing materials during data collection. My thanks also extends to Dr. Ahmadreza Dorosty who guided me from project design to the last. I also acknowledge the Ethics Committee and the Vice Chancellor for Research at Tehran University of Medical Sciences who approved the project in accordance with the tenets of the Helsinki Declaration and the national ethical guideline for medical research (Code IR.TUMS.REC.1394.346). Finally, my thanks goes to the elderly people who participated in this study.

\section{REFERENCES}

1. Delmonico MJ, Harris TB, Lee JS et al. Alternative definitions of sarcopenia, lower extremity performance, and functional impairment with aging in older men and women. J Am Geriatr Soc 2007; 55: 769.

2. Goodpaster BH, Park SW, Harris TB, et al. The loss of skeletal muscle strength, mass, and quality in older adults: The health, aging and body composition study. J Gerontol A Biol Sci Med Sci 2006; 61: 1059-64.

3. Rosenberg R. Epidemiologic and methodological problems in determining nutritional status of older persons. Am J Clin Nutr 1989; 50(5 Suppl):1121-235.

4. Cruz-Jentoft AJ, Baeyens JP, Bauer JM, Boirie Y, Cederholm T, Landi F, et al. Sarcopenia: European consensus on definition and diagnosis: Report of the European Working Group on Sarcopenia in Older People. Age Ageing 2010; 39:412-23.

5. Clark BC, Manini TM. Sarcopenia $\neq$ dynapenia. J Gerontol A Biol Sci Med Sci 2008; 63:829-34. 
6. Muscaritoli M, Anker SD, Argilés J, Aversa Z, Bauer JM, Biolo G, et al. Consensus definition of sarcopenia, cachexia and precachexia: Joint document elaborated by Special Interest Groups (SIG) "cachexiaanorexia in chronic wasting diseases" and "nutrition in geriatrics". Clin Nutr 2010; 29:154-9.

7. Fielding RA, Vellas B, Evans WJ, Bhasin S, Morley JE, Newman AB, et al. Sarcopenia: an undiagnosed condition in older adults. Current consensus definition: prevalence, etiology, and consequences. International Working Group on Sarcopenia. J Am Med Dir Assoc 2011; 12:249-56.

8. Morley JE, Abbatecola AM, Argiles JM, Baracos V, Bauer J, Bhasin S, et al. Society on Sarcopenia, Cachexia and Wasting Disorders Trialist workshop. Sarcopenia with limited mobility: an international consensus. $J \mathrm{Am}$ Med Dir Assoc. 2011; 12:403-9.

9. Chen LK, Liu LK, Woo J, Assantachai P, Auyeung TW, Bahyah KS, et al. Sarcopenia in Asia: consensus report of the Asian Working Group for Sarcopenia. J Am Med Dir Assoc. 2014; 15:95-101.

10. Dam TT, Peters KW, Fragala M, Cawthon PM, Harris TB, McLean R, et al. An evidence-based comparison of operational criteria for the presence of sarcopenia. $J$ Gerontol A Biol Sci Med Sci. 2014; 69:58490.

11. Anker SD, Coats AJ, Morley JE, Rosano G, Bernabei R, von Haehling $S$, et al. Muscle wasting disease: a proposal for a new disease classification. J Cachexia Sarcopenia Muscle. 2014; 5:1-3.

12. Roth SM, Metter EJ, Ling S, Ferrucci L. Inflammatory factors in age-related muscle wasting. Curr Opin Rheumatol 2006; 18:62530.

13. Zoico E, Di Francesco V, Guralnik JM, Mazzali G, Bortolani A, Guariento S, et al. Physical disability and muscular strength in relation to obesity and different body composition indexes in a sample of healthy elderly women. Int $J$ Obes Relat Metab Disord. 2004; 28:234-41.

14. Nass R, Johannsson G, Christiansen JS, Kopchick JJ, Thorner MO. The aging population-is there a role for endocrine interventions? Growth HormIGF Res. 2009; 19:89-100.

15. Chaput JP, Lord C, Cloutier M, Aubertin Leheudre M, Goulet ED, Rousseau S, et al. Relationship between antioxidant intakes and class I sarcopenia in elderly men and women. J Nutr Health Aging. 2007; 11:363-9.

16. Zimmer, V. Prachuabmoh. Comparing the socioeconomic status health gradient among adults 50 and older across rural and urban areas of Thailand in 1994 and 2007; Soc Sci Med.2012; 74:1921-1928.

17. Kagamimori, A. Gaina, A. Nasermoaddeli Socioeconomic status and health in the Japanese population. Soc Sci Med, 68 (2009), pp. 21 52-2160.

18. Ishikawa, H. Nishiuchi, H. Hayashi, K. Viswanath. Socioeconomic status and health communication inequalities in Japan: a nationwide cross-sectional survey PLoS ONE. 2012; 7:1932-1940. Fried LP, Tangen CM, Walston et al. Frailty in older adults: evidence for a phenotype. J Gerontol A Biol Sci Med Sci.2001, 56:M146-156.

19. Kim JS, Wilson JM, Lee SR. Dietary implications on mechanisms of sarcopenia: Roles of protein, amino acids and antioxidants. J Nutr Biochem 2010; 21:1-13.

20. Paddon-Jones D, Rasmussen BB. Dietary protein recommendations and the prevention of sarcopenia. Curr Opin Clin Nutr Metab Care 2009; 12:86-90.

21. Morais JA, Chevalier S, Gougeon R. Protein turnover and requirements in the healthy and frail elderly. J Nutr Health Aging 2006; 10:272-83.

22. Tieland M, Borgonjen-Van den Berg KJ, van Loon LJ, de Groot LC. Dietary protein intake in community-dwelling, frail, and institutionalized elderly people: Scope for improvement. Eur J Nutr 2012; 51:173-9.

23. Stookey JD, Adair LS, Popkin BM. Do protein and energy intakes explain long-term changes in body composition? $J$ Nutr Health Aging 2005; 9:5-17.

24. Houston DK, Nicklas BJ, Ding J, Harris TB, Tylavsky FA, Newman AB, et al. Dietary protein intake is associated with lean mass change in older, community-dwelling adults: The Health, Aging, and Body Composition 
(Health ABC) Study. Am J Clin Nutr 2008; $87: 150-5$.

25. Mithal A, Bonjour JP, Boonen S, Burckhardt P, Degens H, El Hajj Fuleihan G, et al. Impact of nutrition on muscle mass, strength, and performance in older adults. Osteoporosis Int 2013; 24:1555-66.

26. Scott D, Blizzard L, Fell J, Giles G, Jones G. Associations between dietary nutrient intake and muscle mass and strength in communitydwelling older adults: The Tasmanian Older Adult Cohort Study. $J$ Am Geriatr Soc 2010;58:2129-34.

27. Visser M, Deeg DJ, Lips P. Low vitamin D and high parathyroid hormone levels as determinants of loss of muscle strength and muscle mass (sarcopenia): The Longitudinal Aging Study Amsterdam. J Clin Endocr Metab 2003; 88:5766-72.

28. Kaiser M, Bandinelli S, Lunenfeld B. Frailty and the role of nutrition in older people. A review of the current literature. Acta Biomedica 2010; 81(Supp11):37-45.

29. Dawson-Hughes B, Harris SS, Ceglia L. Alkaline diets favor lean tissue mass in older adults. Am J Clin Nutr 2008; 87:662-5.

30. Semba RD, Blaum C, Guralnik JM, Moncrief DT, Ricks MO, Fried LP. Carotenoids and vitamin $\mathrm{E}$ status are associated with indicators of sarcopenia among older women living in the community. Aging Clin Exp Res 2003; $15: 482-7$

31. Gillette-Guyonnet S, Nourhashemi F, Andrieu S, Cantet C, Albarede JL, Vellas B, et al. Body composition in French women $75 \mathrm{p}$ years of age: The EPIDOS study. Mech Ageing Dev 2003; 124:311-316.

32. Topinkova, E. Aging, disability, and frailty. Ann Nutr Metab. 2008; 511-526.

33. Janssen I, Heymsfield SB, Ross R. Low relative skeletal muscle mass (sarcopenia) in older persons is associated with functional impairment and physical disability. $J \mathrm{Am}$ Geriatr Soc. 2002; 50:889-896.

34. Laurentani F, Russo C, Bandinelli $\mathrm{S}$ et al. Age-associated changes in skeletal muscles and their effect on mobility: an operational diagnosis of sarcopenia. Journal of Applied Physiology. 2003, 95:1851-1860.

35. Cesari, M., Kritchevsky, S.B., Newman et al. Added value of physical performance measures in predicting adverse health-related events: results from the health, aging and body composition study. J Am Geriatr Soc. 2009; 57:251-259.

36. Chien MY, Huang TY, Wu YT. Prevalence of sarcopenia estimated using a bioelectrical impedance analysis prediction equation in community-dwelling elderly people in Taiwan.J Am Geriatr Soc. 2008; 56:17101715.

37. Guralnik JM, Ferrucci L, Pieper CF et al. Lower extremity function and subsequent disability: consistency across studies, predictive models, and value of gait speed alone compared with the short physical performance battery. J Gerontol A Biol Sci Med Sci. 2000; 55:M221-231.

38. Lau EM, Lynn HS, Woo JW, Kwok TC, Melton LJ III. Prevalence of and risk factors for sarcopenia in elderly Chinese men and women. J Gerontol A Biol Sci Med Sci. 2005; 60:213-216.

39. Baumgartner RN, Koehler KM, Gallagher D, Romero L, Heymsfield SB, Ross RR, Garry PJ, Lindeman RD. Epidemiology of sarcopenia among the elderly in New Mexico. Am J Epidemiol. 1998; 147:755-763.

40. Wu IC, Lin CC, Hsiung CA et al. Epidemiology of Sarcopenia among community-dwelling older adults in Taiwan: a pooled analysis of broader adoption Sarcopenia assessments, Geriatric Gerontol Int. 2014; 14 supple 1:52-60.

41. Gariballa S, Alessa A. Sarcopenia: Prevalence and prognostic significance in hospitalized patients. Clin Nutr 2013; 32: 772-6.

42. Smoliner C, Sieber CC, Wirth R. Prevalence of Sarcopenia in geriatric hospitalized patients. JAM Med Dir Association. 2014; 15:267-72.

43. Rossi AP, Fantin F, Micciolo R, Bertochi M, Bertassello P, Zanadrea V et al. Identifying Sarcopenia in acute care setting patient. $J$ Am Med Dir Assoc. 2014; 15: 303.e7-e12. 